\title{
Robust Control of a Magnetostrictive Actuator
}

\author{
James M. Nealis ${ }^{1}$ and Ralph C. Smith ${ }^{2}$ \\ Department of Mathematics \\ Center for Research in Scientific Computation \\ North Carolina State University \\ Raleigh, NC 27695
}

\begin{abstract}
Applications utilizing smart materials are rapidly increasing and include high speed milling and hybrid motor design. Such application utilize magnetostrictive transducers operating in hysteretic and nonlinear regimes. To achieve the high performance capabilities of these transducers, models and control laws must accommodate the nonlinear dynamics in a manner which is robust and facilitates real-time implementation. To this end, the models and control algorithms must utilize known physics to the highest degree possible, be low order, and be sufficiently robust to operate under realistic conditions. In this paper we consider the robust control of a smart structure with disturbances due to inherent hysteresis and sensor noise. We demonstrate the techniques on a magnetostrictive transducers but they are sufficiently general to be utilized on several commonly used smart materials. The performance of the control strategies are illustrated through numerical examples.
\end{abstract}

Keywords: Robust control, hysteresis and constitutive nonlinearities, magnetostrictive materials

\section{Introduction}

Smart materials are increasingly utilized for a variety of applications, including tunable lenses, modular antennas, atomic force microscopes, and high speed milling. Materials such as piezoceramics (PZT) and magnetostrictives are ideal for such applications because of their set point accuracy and high bandwidth capabilities. However, these materials exhibit hysteresis and constitutive nonlinearities which must be accommodated to achieve the high performance demands of these applications. At low frequencies and moderate drive levels, these effects can often be mitigated through feedback loops. At high drive levels or high frequencies, however, the hysteresis and nonlinear dynamics must be incorporated into models and subsequent control designs. One method of attenuating the nonlinear hysteretic behavior of these materials is to develop an inverse model. This method requires a hysteresis model which allows an inverse that can be calculated in real-time. This type of inverse filtering has been applied previously employing a domain wall hysteresis model $[4,5]$ as well as Preisach models [10] (for a description of the domain wall hysteresis model, see [1, 2]). In this paper we develop an inverse compensation method which utilizes a free energy hysteresis model. In a realistic application, the effects of hysteresis are not the only disturbances to be overcome. There is a certain amount of noise associated with any sensing device. This noise will be fed into the controller and can greatly decrease the closed loop performance. In this paper, we consider the development of a robust feedback control law which is able to reject the disturbances due to sensor noise and the hysteretic behavior.

To illustrate, we will consider the prototypical magnetostrictive actuator depicted in Figure 1. Input stresses and displacements are provided by the Terfenol-D rod in response to fields generated by the surrounding solenoid.

\footnotetext{
${ }^{1}$ Email: jmnealis@unity.ncsu.edu, Telephone: (919) 515-8968

${ }^{2}$ Email: rsmith@eos.ncsu.edu; Telephone: (919) 515-7552
} 


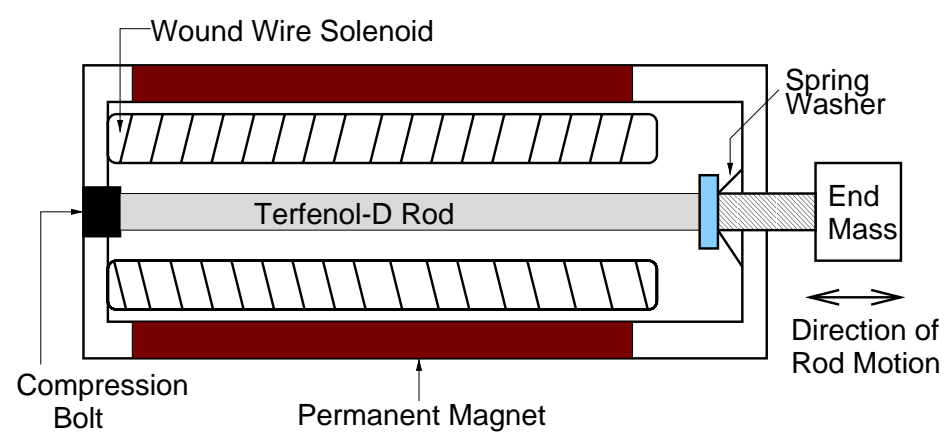

Figure 1. Terfenol-D transducer.

The structure is encased in a permanent magnet which provides a biasing field to obtain bi-directional strains and a mechanism for flux shaping in the transducer. As detailed in [2], such actuators have the capability of generating broadband, high force, responses. However, they also exhibit varying degrees of hysteresis and nonlinearities in the relation between the input field $H$ and the magnetization $M$ in the Terfenol-D rod. We concentrate on a magnetostrictive transducer in this paper but note that the hysteresis model and control design presented are sufficiently general to permit direct extension to analogous piezoelectric and ferroelectric models as developed in $[7,8,9]$.

\section{Free Energy Hysteresis Model}

In this section we shall summarize the free energy hysteresis model presented in [6]. This model is based on the quantification of energy required to reorient dipoles in combination with stochastic homogenization techniques to accommodate variations in coercive and effective fields. For this development, we assume fixed temperatures. The model also ignores losses due to eddy currents and therefore should be employed for low frequency drive levels. A further assumption is that the spring washer in the transducer provides enough prestress to dominate the crystal anisotropies (see Figure 1).

We first consider the Helmholtz energy which incorporates the internal energy due to the interaction of dipole moments. Under the assumption that dipoles orient either with the applied field or diametrically opposite, it is illustrated in [6] that a reasonable form of the Helmholtz energy is

$$
\psi(M)= \begin{cases}\frac{1}{2} \eta\left(M+M_{R}\right)^{2} & , M \leq-M_{I} \\ \frac{1}{2} \eta\left(M-M_{R}\right)^{2} & , M \geq M_{I} \\ \frac{1}{2} \eta\left(M_{I}-M_{R}\right)\left(\frac{M^{2}}{M_{I}}-M_{R}\right) & ,|M|<M_{I} .\end{cases}
$$

As depicted in Figure 2, $M_{R}$ and $M_{I}$ respectively denote the point at which the minimum of $\psi$ occurs and the inflection point.

In the presence of an applied field $H$, the Gibbs energy is given by

$$
G=\psi-H M
$$

Note that the magnetostatic energy is $\varepsilon=\mu_{0} H M$ where $\mu_{0}$ denotes the magnetic permeability. The Gibbs free energy given in (2) can be interpreted as incorporating $\mu_{0}$ into $\psi$ for simplicity.

For a homogeneous material with the effective field taken as the applied field, the local average magnetization can be expressed as

$$
\bar{M}=x_{+}\left\langle M_{+}\right\rangle+x_{-}\left\langle M_{-}\right\rangle
$$



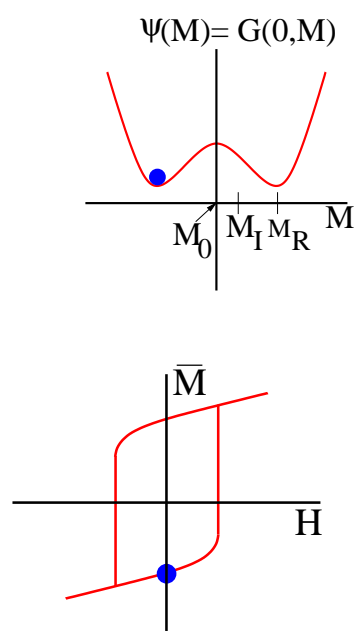

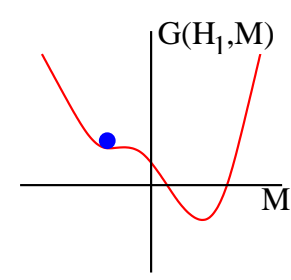

(a)

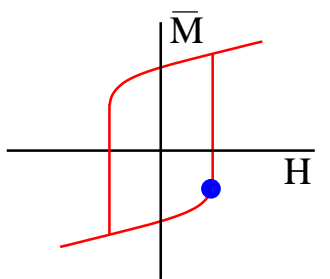

(b)
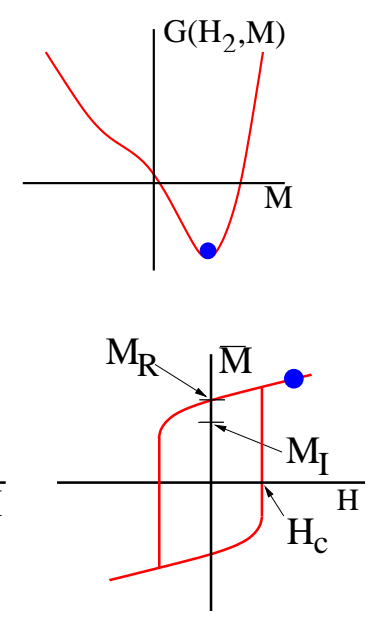

Figure 2. (a) Helmholtz energy $\psi$ and Gibbs energy for increasing field $H$. (b) Local magnetization $\bar{M}$ for increasing field $H$.

where $x_{+}$and $x_{-}$denote the fraction of moments having the positive or negative orientation, respectively, and $\left\langle M_{+}\right\rangle$and $\left\langle M_{-}\right\rangle$are the expected values of the magnetization for moments in the positive or negative orientation respectively. As detailed in [6], $\left\langle M_{+}\right\rangle$is quantified by

$$
\left\langle M_{+}\right\rangle=\frac{\int_{M_{I}}^{\infty} M e^{-G(H, M) V / k T} d M}{\int_{M_{I}}^{\infty} e^{-G(H, M) V / k T} d M} .
$$

Here $e^{-G(H, M) V / k T}$ represents the probability of obtaining an energy level $G$ and the denominator of (4) assures that the probability of integrating over all possible magnetization values is 1 . Here $V$ is the lattice volume, $k$ is Boltzman's constant and $T$ is the temperature. The relation for $\left\langle M_{-}\right\rangle$is given similarly as

$$
\left\langle M_{-}\right\rangle=\frac{\int_{-\infty}^{M_{I}} M e^{-G(H, M) V / k T} d M}{\int_{-\infty}^{M_{I}} e^{-G(H, M) V / k T} d M} .
$$

The moment fraction are quantified by the evolution equations

$$
\begin{aligned}
& \dot{x}_{+}=-p_{+-} x_{+}+p_{-+} x_{-} \\
& \dot{x}_{-}=-p_{-+} x_{-}+p_{+-} x_{+}
\end{aligned}
$$

where $p_{+-}$and $p_{-+}$are the likelihoods of switching form positive to negative orientation and switching from negative to positive orientation, respectively. They are given as

$$
\begin{gathered}
p_{+-}=\sqrt{\frac{k T}{2 \pi m}} \frac{e^{-G\left(H, M_{0}\right) V / k T}}{\int_{M_{I}}^{\infty} e^{-G(H, M) V / k T} d M} \\
p_{-+}=\sqrt{\frac{k T}{2 \pi m}} \frac{e^{-G\left(H,-M_{0}\right) V / k T}}{\int_{-\infty}^{-M_{I}} e^{-G(H, M) V / k T} d M}
\end{gathered}
$$

where $m$ is the mass of lattice volume $V$. The relation between the applied field $H$ and the magnetization $M$ exhibits both hysteresis and nonlinear transition because the local magnetization (3) is probabilistic. The steepness of the transition depend on the ratio of $G V$ to $k T$.

We will now describe an alternative formulation of the magnetization which we will employ numerically. We 


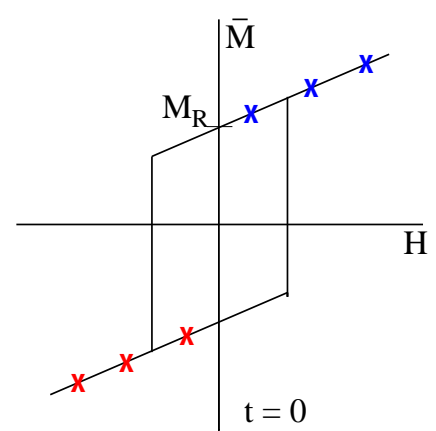

(a)

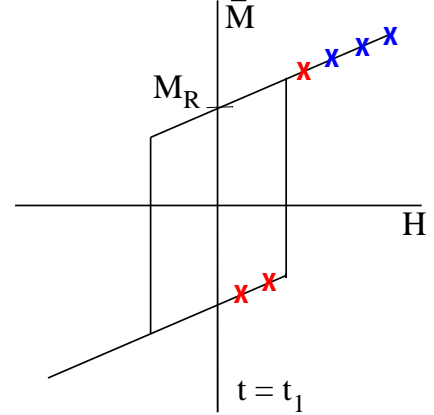

(b)

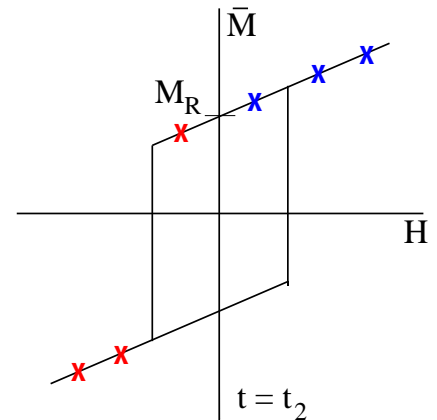

(c)

Figure 3. Varying field levels for the limiting case local average magnetization $\bar{M}$.

can analyze the equilibrium behavior after the moments have switched by examining the equilibrium condition $\frac{\partial G}{\partial M}=0$ which yields $H=\frac{\partial \psi}{\partial M}$. Therefore the local model predicts a linear relationship between $H$ and $\bar{M}$ with a slope of $\frac{1}{\eta}$. We can employ asymptotic relations to simplify the magnetization and consider the jumps in magnetization to occur instantaneously. As detailed in [6], the local magnetization in this limiting case is given as

$$
\left[\bar{M}\left(H ; H_{c}, \xi\right)\right](t)= \begin{cases}{\left[\bar{M}\left(H ; H_{c}, \xi\right)\right](0)} & , \tau(t)=\varnothing \\ \frac{H}{\eta}-M_{R} & , \tau(t) \neq \varnothing \text { and } H(\max \tau(t))=-H_{c} \\ \frac{H}{\eta}+M_{R} & , \tau(t) \neq \varnothing \text { and } H(\max \tau(t))=H_{c}\end{cases}
$$

where the transition points are specified as

$$
\tau(t)=\left\{t \in\left(0, T_{f}\right] \mid H(t)=-H_{c} \text { or } H(t)=H_{c}\right\}
$$

and the initial moment orientation is given as

$$
\left[\bar{M}\left(H ; H_{c}, \xi\right)\right](0)=\left\{\begin{array}{l}
\frac{H}{\eta}-M_{R} \quad, H(0) \leq-H_{c} \\
\xi \quad,-H_{c}<H(0)<H_{c} \\
\frac{H}{\eta}+M_{R} \quad, H(0) \geq H_{c}
\end{array}\right.
$$

Figure 3 depicts the progression of local average magnetization points for varying applied field levels.

The relation (10) is derived under the assumption that the lattice structure is homogeneous and hence the domain structure is homogeneous. This implies that the free energy profile for the different regions of the Terfenol-D are identical. This assumption is overly simple since it ignores material defects, polycrystallinity and nonuniformities in the crystals. Also, the model has assumed that the effective field at the domain level is the applied field. To extend the local model, stochastic distributions are incorporated to develop a bulk magnetization model for a nonhomogeneous Terfenol-D sample with nonconstant effective fields (see [6] for details).

We shall begin by summarizing the inclusion of a distribution of free energy profile to accommodate material nonhomogeneities (see Figure 4). The nonhomogeneities are included by assuming the parameters $M_{R}$ and $M_{I}$, or equivalently $H_{c}=\eta\left(M_{R}-M_{I}\right)$, are normally distributed with mean $\bar{H}_{c}$. The total magnetization can then be given as

$$
M(H)=\int_{0}^{\infty} \bar{M}(H ; H c, \xi) f\left(H_{c}\right) d H_{c}
$$

with density

$$
f\left(H_{c}\right)=C_{1} e^{-\left(H_{c}-\bar{H}_{c}\right)^{2} / b} .
$$

The parameters $C_{1}$ and $b$ are positive and $\bar{M}$ is given by (3) or (10).

The second extension entails the incorporation of variations in the effective field. The effective field is assumed 

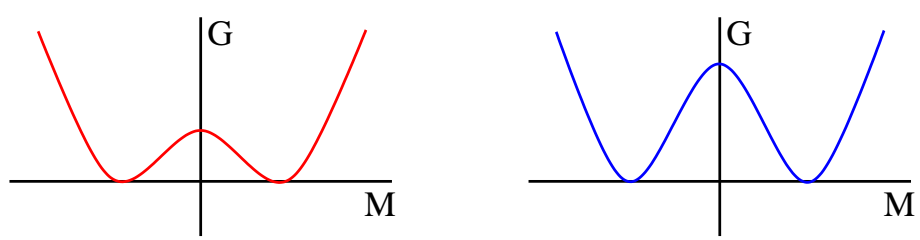

(a)
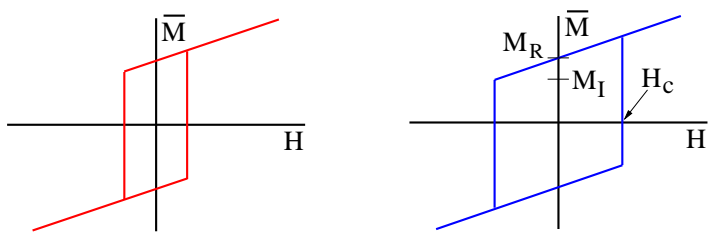

(b)

Figure 4. (a) Free energies for nonhomogeneous domain structure. (b) Resulting variations in the local coercive field $H_{c}$.

to be normally distributed with mean $\mathcal{H}$. Therefore, for a fixed coercive field, the magnetization can be given as

$$
M(H)=\int_{-\infty}^{\infty} \bar{M}\left(H ; H_{c}, \xi\right) C_{2} e^{-(H-\mathcal{H})^{2} / \bar{b}} d \mathcal{H} d H_{c} .
$$

Combining coercive field and effective field distributions for the full magnetization of a nonhomogeneous polycrystalline sample of Terfenol-D with variable effective fields yields the magnetization relation

$$
M(H)=C \int_{0}^{\infty} \int_{-\infty}^{\infty}\left[\bar{M}\left(\mathcal{H}+H ; H_{c}, \xi\right)\right](t) e^{\mathcal{H}^{2}} e^{-\left(H_{c}-\bar{H}_{c}\right)^{2} / b} d \mathcal{H} d H_{c}
$$

For numerical implementation of the free energy hysteresis model, the distributions for the effective field and coercive field are evaluated using a composite quadrature rule. This allows the hysteresis model to be computed algebraically and hence at low cost. Because of the exponential decay of the distributions, they can be truncated to speed computation. The model is sufficiently simple to allow the possibility of real time implementation and has a relatively low number of material dependent parameters.

\section{Inverse Hysteresis Model}

To construct an inverse compensator, the monotonicity of the hysteresis model is exploited. To determine the magnetic field required to create a desired magnetization, the hysteresis model is advanced until the desired magnetization is surpassed. Then the magnetic field is computed by a linear interpolation between the last two points. The computational speed of the inverse compensator depends on the size of the step taken in advancing the hysteresis model. Larger steps will increase the speed while decreasing the accuracy of the inverse compensator. To facilitate real time control, the control method must be designed to reject a significant amount of error in the linearization of the hysteresis. The linearization error for an input signal with a frequency of $1 \mathrm{~Hz}$ and a step size of $\Delta H=1$ employed in the inverse model is plotted in Figure 5a. While the error appears quite large the relative error, illustrated in Figure 5b, is reasonable. The control design must be able to reject this error to the input of the plant.

\section{Full Actuator Model}

We have described the model for the magnetization in the Terfenol-D sample due to an applied field. We must next quantify the strains, forces, and displacements generated by these changes in magnetization for the 


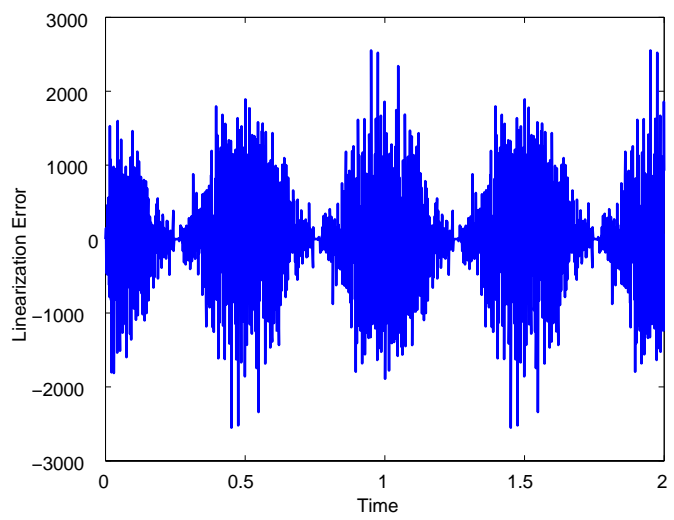

(a)

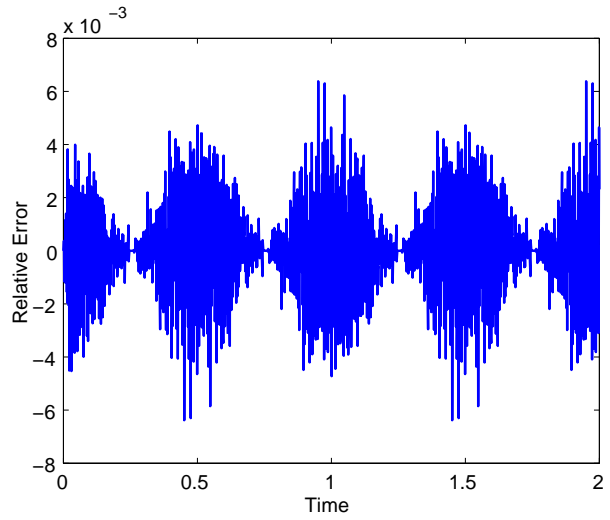

(b)

Figure 5. (a) Hysteresis linearization error and (b) relative error.

prototypical transducer under consideration (see Figure 1). The model for the transducer is based on the model given in [2].

One end of the rod $(x=0)$ is assumed to be fixed while the other end $(x=L)$ is constrained by a damped oscillator and has an attached point mass (see Figure 6). The internal damping coefficient, density, Young's Modulus and point mass are given by $c_{D}, \rho, E$, and $M_{L}$ respectively. The damping spring has stiffness $k_{L}$ and Kelvin-Voigt damping coefficient $c_{L}$.

Under the assumption of linear elasticity, Kevin-Voigt damping and small displacements, the stress at any point $x, 0 \leq x \leq L$, is given as

$$
\sigma(t, x)=E \frac{\partial w}{\partial x}(t, x)+c_{D} \frac{\partial^{2} w}{\partial x \partial t}(t, x)-E \frac{\lambda_{s}}{M_{s}} M(t, x)^{2}
$$

where $w(t, x)$ is the displacement and $\lambda_{s}$ and $M_{s}$ respectively denote the saturation free strain and saturation magnetization of the Terfenol-D. Balancing forces yields

$$
\rho A \frac{\partial^{2} w}{\partial t^{2}}=\frac{\partial N_{t o t}}{\partial x}
$$

where the resultant is specified by

$$
N_{t o t}(t, x)=E A \frac{\partial w}{\partial w}(t, x)+c_{D} A \frac{\partial^{2} w}{\partial x \partial t}(t, x)-E A \frac{\lambda_{s}}{M_{s}} M(t, x)^{2} .
$$

To obtain appropriate boundary conditions, we first note $w(t, 0)=0$. Balancing forces at $x=L$ yields

$$
N_{t o t}(t, L)=-k_{L} w(t, L)-c_{L} \frac{\partial w}{\partial t}(t, L)-M_{L} \frac{\partial^{2} w}{\partial x \partial t}(t, L) .
$$

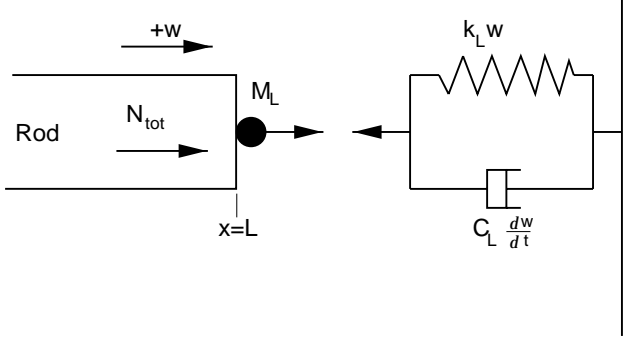

Figure 6. Rod model. 


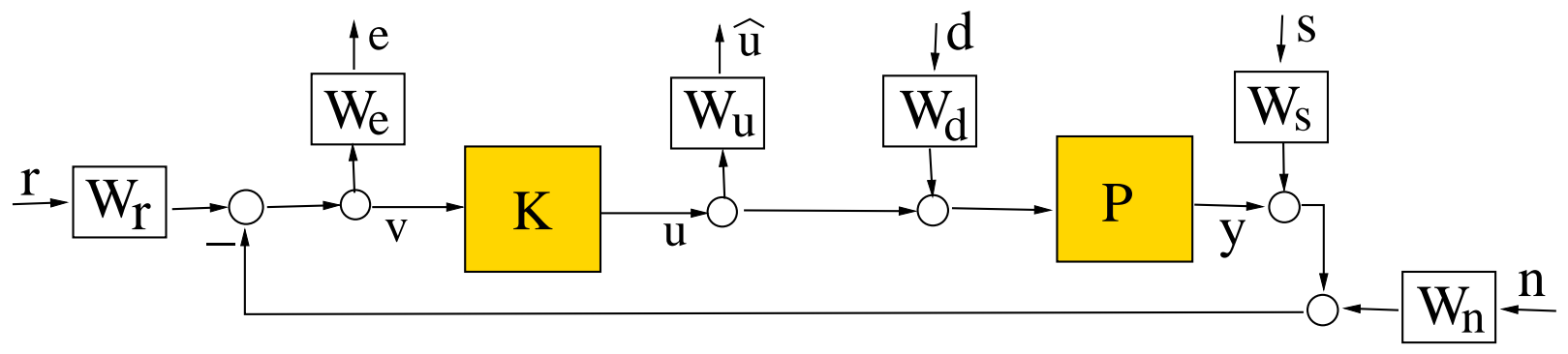

Figure 7. Robust control diagram.

Initial conditions are taken to be $w(0, x)=0$ and $\frac{\partial w}{\partial x}(0, x)=0$.

The model can be implemented utilizing the weak form and a Galerkin finite element approximation. To further simplify our system, we assert that the magnetization of the Terfenol-D rod can be taken as uniform over the length of the rod. This is reasonable since, in present actuator design, flux shaping via the surrounding magnet can be used to minimize end effects in the rod. Hence motion of the tip of the Terfenol-D rod can be modeled by an ordinary differential equation. This observation can be supported by the fact that, since each section of the rod reacts identically to the uniform magnetic field, the elements act uniformly and therefore the transducer dynamics may be modeled as a damped spring-mass. The ODE model we employ for the displacement of the tip of the Terfenol-D rod due to the magnetization is given as

$$
\begin{gathered}
m \ddot{x}+k \dot{x}+c x=\omega M^{2}(t) . \\
x(0)=x_{0} \quad \dot{x}(0)=\dot{x}_{0} .
\end{gathered}
$$

Here the scalars $m, k, c$, and $\omega$ are determined by fitting the model (21) to the Galerkin approximation of (18) or to data from the physical device.

Next we shall linearize the magnetostrictive relationship about a biasing magnetization level of $M_{s} / 2$. This bias can be achieved my means of the permanent magnet (see Figure 1). The dynamics of the Terfenol-D transducer can be now represented by

$$
\begin{gathered}
\ddot{x}+\widehat{k} \dot{x}+\widehat{c} x=\widehat{\omega} M(t) \\
x(0)=x_{0} \quad \dot{x}(0)=\dot{x}_{0} .
\end{gathered}
$$

where $\widehat{k}=k / m=7.8899 \times 10^{3}, \widehat{c}=c / m=6.4251 \times 10^{7}$ and $\widehat{\omega}=1.3724 \times 10^{-2}$. Note that the hysteresis inherent in the Terfenol-D rod is still present in (22) by means of the hysteretic relationship between $H$ and $M$.

\section{Robust Control Design}

In this section, we present a control design which incorporates the presence of external disturbances and minimizes their effects with respect to a given norm. We shall consider the $H_{2}$ norm defined by

$$
\|T\|_{2}^{2}=\frac{1}{2 \pi} \int_{-\infty}^{\infty} \operatorname{trace}\left[T^{*}(j \omega) T(j \omega)\right] d \omega .
$$

The $H_{2}$ norm is chosen because it provides a measure of energy in the system. The inverse compensator developed in Section 3 will be employed to attenuate the hysteresis inherent in the Terfenol-D transducer. Figure 7 shows the block diagram of the system to be controlled. In the diagram $P$ represents the transducer model given by the differential equation (22). The signal to be tracked is denoted by $r$ while the position of the tip of the Terfenol-D rod is denoted by $y$. The signal $d$ represents the error in the linearization of the input by the inverse filter (see Figure 8). The signals $n$ and $s$ represent noise in the sensor the measurements of $y$. For the purposes of this paper, we assume sensor noise with a frequency of $60 \mathrm{~Hz}$. This noise is represented by $s$. The signal $n$ represents higher frequency noise which could be attributed to the sensing device, other external disturbances or, in a 


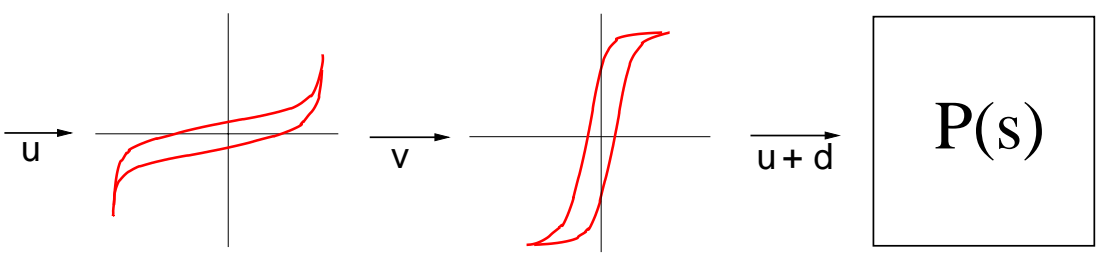

Figure 8. Inverse compensation.

numerical simulation, numerical noise. We have chosen to separate these two signals as we wish to weight them independently. The output signals $e$ and $\widehat{u}$ denote the weighted tracking error and weight output of the controller $K$, respectively. Here $W_{u}, W_{d}, W_{e}, W_{r}, W_{s}$ and $W_{n}$ are weighting functions which are chosen to maximize the performance of the controller utilizing a priori knowledge of the characteristics of the signals.

We can now represent the transfer functions of the open loop system. The maps from the inputs, $r, d, s$ and $n$, to the outputs $\widehat{u}, e$ and $v$ are given as

$$
\begin{gathered}
v=W_{r}[r]-\left(P\left[W_{d}[d]+u\right]+W_{n}[n]+W_{s}[s]\right)=W_{r}[r]-P\left[W_{d}[d]\right]-W_{n}[n]-W_{s}[s]-P[u] \\
e=W_{e}[v] \\
\widehat{u}=W_{u}[u] .
\end{gathered}
$$

Denoting the transfer function matrix from the inputs to the outputs as $G$, we then have

$$
G=\left[\begin{array}{ccccc}
W_{e} W_{r} & -W_{e} P W_{d} & -W_{e} W_{n} & -W e W s & -W_{e} P \\
0 & 0 & 0 & 0 & W_{u} \\
W r & -P W_{d} & -W_{n} & -W_{s} & -P
\end{array}\right]
$$

The system can be represented as a linear fractional transformation as shown in Figure 9. The goal is to design a controller $K$ so the $H_{2}$ norm of transfer function from the inputs to the outputs of our closed loop system, denoted $T$, is minimal.

The open loop system can be partitioned as

$$
G(s)=\left[\begin{array}{c|cc}
A & B_{1} & B_{2} \\
\hline C_{1} & 0 & D_{12} \\
C_{2} & D_{21} & 0
\end{array}\right]
$$

where

$$
G_{11}=\left[\begin{array}{c|c}
A & B_{1} \\
\hline C_{1} & 0
\end{array}\right], \quad G_{12}=\left[\begin{array}{c|c}
A & B_{2} \\
\hline C_{1} & D_{12}
\end{array}\right], \quad G_{21}=\left[\begin{array}{c|c}
A & B_{1} \\
\hline C_{2} & D_{21}
\end{array}\right], \quad G_{22}=\left[\begin{array}{c|c}
A & B_{2} \\
\hline C_{2} & 0
\end{array}\right]
$$

represent the transfer functions from $w$ to $z, u$ to $z, w$ to $v$, and $u$ to $v$, respectively (see Figure 9). We make the following assumptions on our system:

1. $\left(A, B_{1}\right)$ is controllable and $\left(C_{1}, A\right)$ is observable.

2. $\left(A, B_{2}\right)$ is stabilizable and $\left(C_{2}, A\right)$ is detectable.

3. $D_{12}^{*} D_{12}>0$ and $D_{21} D_{21}^{*}>0$.

4. $\left[\begin{array}{cc}A-j \omega I & B_{2} \\ C_{1} & D_{12}\end{array}\right]$ has full column rank for all $\omega$.

5. $\left[\begin{array}{cc}A-j \omega I & B_{1} \\ C_{2} & D_{21}\end{array}\right]$ has full row rank for all $\omega$. 


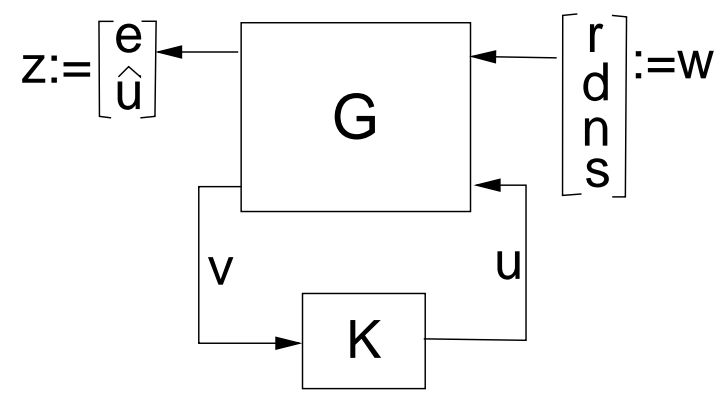

Figure 9. Linear fractional transformation (LFT) representation of transducer.

\subsection{Weighting Functions}

The majority of effort in designing a robust controller lies in the choice of the weighting functions. A discussion on the choice of these function can be found in $[3,11]$. The frequency of the noise $s$ present can be accurately determined for the device measuring the position of the tip of the Terfenol-D rod. The weight $W_{s}$ is chosen to heavily weight frequencies in a bandwidth around our expected noise frequency and attach low weights to frequencies above and below our specified bandwidth. For the simulation, a noise frequency of $60 \mathrm{~Hz}$ is added to the system and $W_{s}$ is taken to be a ninth-order pass-band filter with a bandwidth of $10 \mathrm{~Hz}$ centered at $60 \mathrm{~Hz}$. A higher order filter can be used if a steeper rolloff outside of the frequency band is desired although we have chosen to limit the number of states in the state space representation. The frequency response of $W_{s}$ is illustrated in Figure 10a.

To weight high frequency noise, $W_{n}$ is taken to be a high pass filter. The sixth-order filter, plotted in Figure 10b, is employed in the numerical simulations. The weight $W_{r}$ is taken as a sixth-order pass-band filter. Since the frequency of the reference signal $r$ is known, we can design $W r$ to have a narrow bandwidth which is taken as $1 \mathrm{~Hz}$ centered at $1 \mathrm{~Hz}$. The frequency response of $W_{r}$ is shown in Figure 11.

To determine the weighting function $W_{d}$, a signal with the same frequency as the reference signal was filtered by the inverse compensator and then fed into the free energy hysteresis model. The output is defined to be the desired control signal plus the disturbance $d$ (see Figure 8). The Fourier transform of the disturbance is analyzed to determine $W_{d}$. Figure 12a illustrates the power spectrum of the disturbance. From Figure 12a, it is clear that $W_{d}$ should weight frequencies between 100 and $350 \mathrm{~Hz}$ heavily. Therefore $W_{d}$ is taken as a pass-band filter with a bandwidth of 250 centered at $225 \mathrm{~Hz}$. The frequency response of $W_{d}$ is shown in Figure 12b.

The weighting function on the error signal was taken to be $W_{e}=\frac{\gamma_{e}}{s+\epsilon_{e}}$ with $\gamma_{e}=3.2 \times 10^{6}$ and $\epsilon_{e}=1 \times 10^{-8}$.

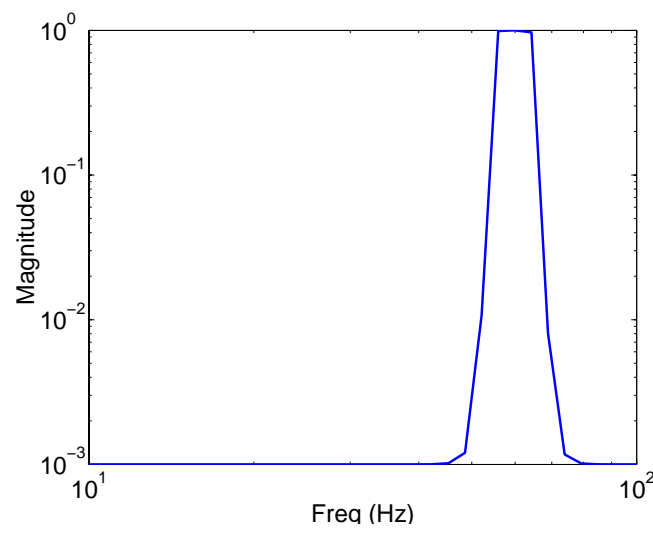

(a)

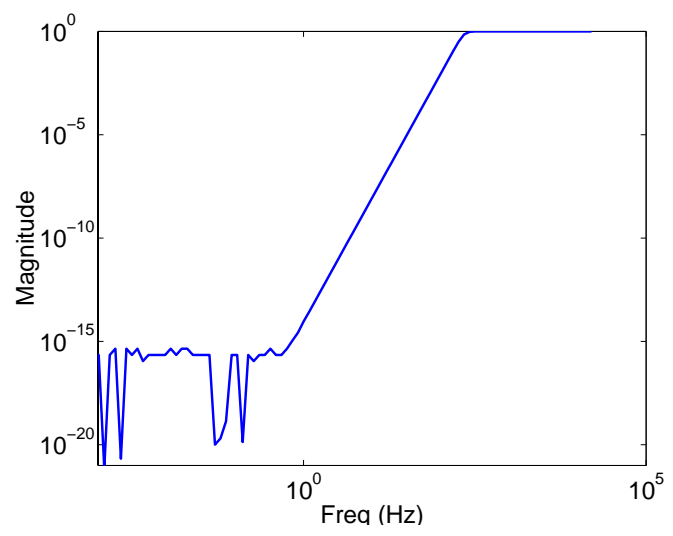

(b)

Figure 10. Frequency response of (a) $W_{s}$ and (b) $W_{n}$. 


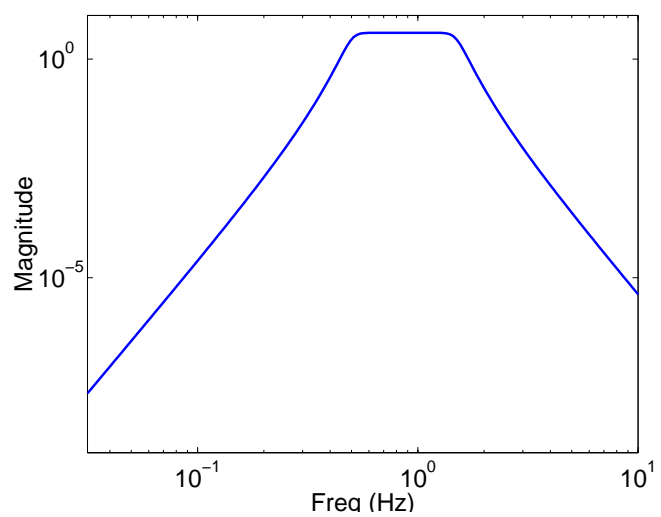

Figure 11. Frequency response of $W_{r}$.

An integrator was chosen to prevent the error from achieving steady state at a nonzero value and the pole was shifted slightly off zero to ensure the controller designed is realizable. We take the weighting function on the controller output to be $W_{u}=5 \times 10^{-6}$. Since we do not experience any problems with saturation, or other such effects, we minimally weight $\widehat{u}$ to focus the controller on tracking and disturbance rejection.

\section{$5.2 \mathrm{H}_{2}$ Optimal Control Design}

Employing the notation defined in (27), the design of the optimal $H_{2}$ controller incorporates two Hamiltonian matrices

$$
H_{2}=\left[\begin{array}{cc}
A-B_{2} R_{1}^{-1} D_{12}^{*} C_{1} & -B_{2} R_{1}^{-1} B_{2}^{*} \\
-C_{1}^{*}\left(I-D_{12} R_{1}^{-1} D_{12}^{*}\right) C_{1} & -\left(A-B_{2} R_{1}^{-1} D_{12}^{*} C_{1}\right)^{*}
\end{array}\right]
$$

and

$$
J_{2}=\left[\begin{array}{cc}
\left(A-B_{2} R_{1}^{-1} D_{12}^{*} C_{1}\right)^{*} & -C_{2}^{*} R_{2}^{-1} C_{2} \\
-B_{1}\left(I-D_{21}^{*} R_{2}^{-1} D_{21}\right) B_{1}^{*} & -\left(A-B_{2} R_{1}^{-1} D_{12}^{*} C_{1}\right)
\end{array}\right]
$$

where $R_{1}=D_{12}^{*} D_{12}>0$ and $R_{2}=D_{21} D_{21}^{*}>0$.

These Hamiltonian matrices give rise to two Riccati equations

$$
\left(A-B_{2} R_{1}^{-1} D_{12}^{*} C_{1}\right)^{*} X_{2}+X_{2}\left(A-B_{2} R_{1}^{-1} D_{12}^{*} C_{1}\right)+X_{2}\left(-B_{2} R_{1}^{-1} B_{2}^{*}\right) X_{2}-C_{1}^{*}\left(I-D_{12} R_{1}^{-1} D_{12}^{*}\right) C_{1}
$$

and

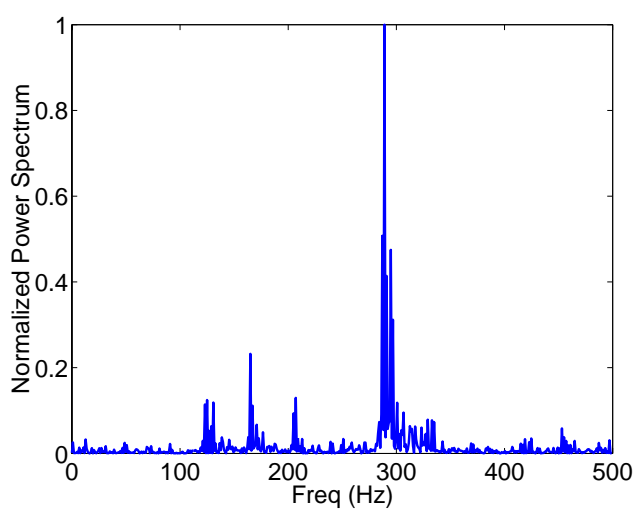

(a)

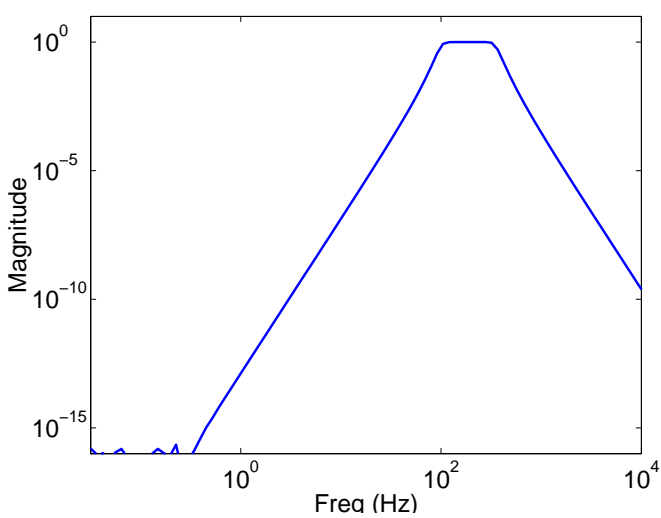

(b)

Figure 12. (a) Power spectrum of $d$, and (b) frequency response of $W_{d}$. 


$$
\left(A-B_{2} R_{1}^{-1} D_{12}^{*} C_{1}\right) Y_{2}+Y_{2}\left(A-B_{2} R_{1}^{-1} D_{12}^{*} C_{1}\right)^{*}+Y_{2}\left(-C_{2}^{*} R_{2}^{-1} C_{2}\right) Y_{2}-B_{1}\left(I-D_{21}^{*} R_{2}^{-1} D_{21}\right) B_{1}^{*} .
$$

Now that the transducer is represented and the Riccati equations have been defined, the following theorem from [11], guaranteeing an $H_{2}$ optimal controller, can be employed.

Theorem 1: There exists a unique controller which minimizes the $H_{2}$ norm of the closed loop system if:

1. $H_{2} \in \operatorname{dom}\left(\right.$ Ric) and $X_{2}:=\operatorname{Ric}\left(H_{2}\right)>0$

2. $J_{2} \in \operatorname{dom}(\operatorname{Ric})$ and $Y_{2}:=\operatorname{Ric}\left(J_{2}\right)>0$

The $H_{2}$ optimal controller is given by

$$
K \equiv\left[\begin{array}{c|c}
A_{2} & -L_{2} \\
\hline F_{2} & 0
\end{array}\right]
$$

where

$$
A_{2} \equiv A+B_{2} F_{2}+L_{2} C_{2}, \quad F_{2} \equiv-R_{1}^{-1}\left(B_{2}^{*} X_{2}+D_{12}^{*} C_{1}\right), \quad L_{2} \equiv-\left(Y_{2} C_{2}^{*}+B_{1} D_{21}^{*}\right) R_{2}^{-1} .
$$

\subsection{Numerical Results}

The numerical results for the $\mathrm{H}_{2}$ robust control law are presented in this section. The results were computed using the noise signal $s$ with a magnitude of $1 \times 10^{-5}$ and a frequency of $60 \mathrm{~Hz}$. The inverse compensator was computed using 4 quadrature points and 20 divisions for the coercive field and 40 divisions for the effective field. A step size of $\Delta H=1$ was employed for the hysteresis inverse model. These parameters ensure a simulation of the control process which has the potential for real-time implementation. The tracking performance with the presence of sensor noise and the disturbance is illustrated in Figure 13. Figure 13a illustrates the controller's ability to track the reference signal and reject the noise and disturbance signals. A tracking error less that 2 microns is achieved after a short period, as illustrated in Figure 13b, and the noise signals and effects of the inverse compensation for the hysteresis are effectively attenuated.

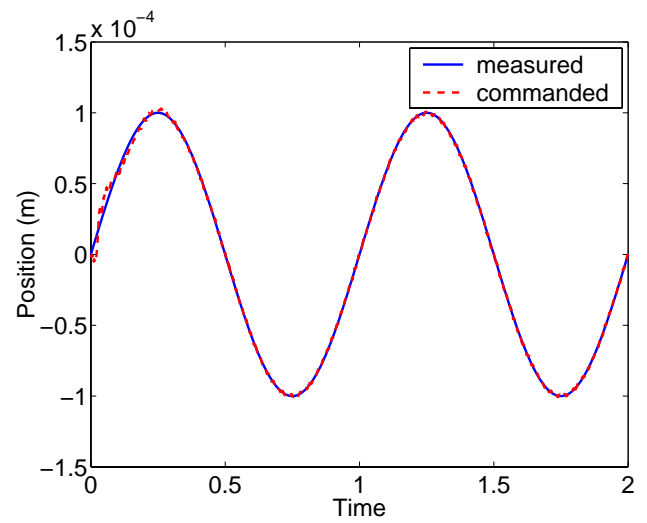

(a)

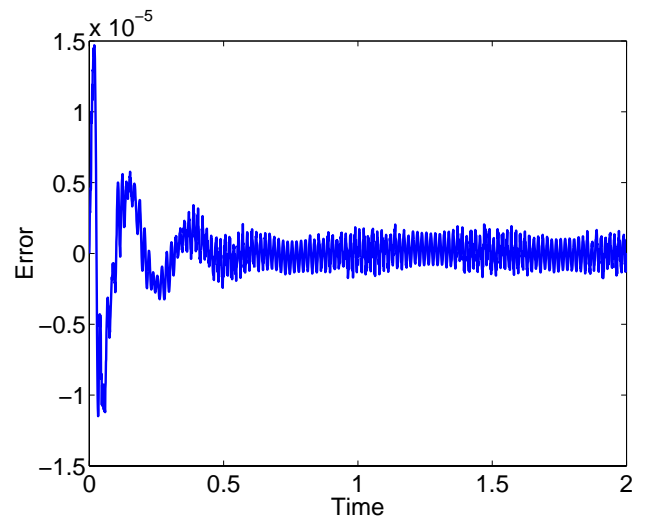

(b)

Figure 13. $H_{2}$ (a) tracking performance and (b) tracking error. 


\section{Conclusions}

This paper has outlined an $\mathrm{H}_{2}$ robust control design for a smart system. Although the methods were illustrated on a magnetostrictive transducer, they can be applied to systems utilizing piezoceramic or shape memory alloys as well. It was shown that an $H_{2}$ control design was capable of maintaining accurate tracking while rejecting sensor noise and a disturbance due to the inexact inverse filter. The same methodology can be used to include other disturbances to the system if needed. Details were provided regarding the choice of weighting functions, or filters, since this step in the robust control formulation is crucial to achieving the balance between tracking requirements and disturbance rejection needed for a particular application.

\section{Acknowledgments}

This research was supported in part by the Air Force Office of Scientific Research under the grant AFOSRF49620-01-1-0107.

\section{References}

[1] F.T. Calkins, R.C. Smith and A.B. Flatau, "An Energy-based Hysteresis Model for Magnetostrictive Transducers," IEEE Transactions on Magnetics, 36(2), pp. 429-439, 2000.

[2] M.J. Dapino, R.C. Smith and A.B. Flatau, "A Structural Strain Model for Magnetostrictive Transducers," IEEE Transactions on Magnetics, 36(3), pp. 545-556, 2000.

[3] D. K. Lindner, Introduction to Signals and Systems, McGraw-Hill, New York, 1999.

[4] J. Nealis and R.C. Smith, "Partial Inverse Compensation Techniques for Linear Control Design in Magnetostrictive Transducers," Proceedings of the SPIE, Smart Structures and Materials, 2001, Vol. 4326, pp. 462$473,2001$.

[5] R.C. Smith, C. Bouton and R. Zrostlik, "Partial and Full Inverse Compensation for Hysteresis in Smart Material Systems," Proceedings of the 2000 American Control Conference.

[6] R.C. Smith, M.J. Dapino and S. Seelecke, "A Free Energy Model for Hysteresis in Magnetostrictive Transducers," Journal of Applied Physics, 93(1), pp. 458-466, 2003.

[7] R.C. Smith and C.L. Hom, "A Domain Wall Theory for Ferroelectric Hysteresis," Journal of Intelligent Material Systems and Structures, 10(3), pp. 195-213, 1999.

[8] R.C. Smith and J.E. Massad, "A Unified Methodology for Modeling Hysteresis in Ferroelectric, Ferromagnetic and Ferroelastic Materials," Proceedings of DETC'01 the ASME Design Engineering Technical Conference and Computers and Information in Engineering Conference, Vol. 6, Pt. B, pp. 1389-1398, 2001.

[9] R.C. Smith and Z. Ounaies, "A Domain Wall Model for Hysteresis in Piezoelectric Materials," Journal of Intelligent Material Systems and Structures, 11(1), pp. 62-79, 2000.

[10] G. Tao and P. V. Kokotovic, Adaptive Control of Systems with Actuator and Sensor Nonlinearities, John Wiley and Sons, New Jersey, 1996.

[11] K. Zhou and J.C. Doyle, Essentials of Robust Control, Prentice Hall, New Jersey, 1998. 